\title{
Contacto elastoplástico entre superficies rugosas con distribución de Gauss y exponencial
}

\author{
Elastic-plastic Contact between Rough Surfaces \\ with Gauss and Exponential Distribution
}

\author{
Sánchez-Rodríguez A. \\ Centro de Ingeniería y Desarrollo Industrial (CIDESI) \\ Correo:asanchez@itc.mx \\ Echeverría-Villagómez J.S. \\ Instituto Tecnológico de Celaya \\ Correo:saleche@cenam.mx \\ Lesso-Arroyo R. \\ Departamento de Ingeniería Mecánica \\ Instituto Tecnológico de Celaya \\ Correo:rlesso@itc.mx
}

\author{
García-Rodríguez F.J. \\ Departamento de Ingeniería Mecánica \\ Instituto Tecnológico de Celaya \\ Correo:fcojav@itc.mx \\ Salas-Zuñiga R. \\ Centro de Desarrollo y Tecnología (CIDET) \\ Correo:mide2004@prodigy.net.mx,Cenit2005@prodigy.net.mx
}

Información del artículo: recibido: junio de 2009, reevaluado: febrero de 2011, aceptado: junio de 2011

\section{Resumen}

Para modelar el contacto elastoplástico de superficies rugosas se analiza la función de distribución probabilística exponencial de la altura de las asperidades propuesta por Greenwood y Williamson (1966) como una aproximación de la distribución de Gauss, y se encuentra que es poco precisa para la mayoría de los casos. Se aplica la función de distribución probabilística exponencial modificada propuesta por Polycarpou y Etsión (1999) al modelo reciente de contacto elastoplástico de Kogut y Etsion (2004), con el propósito de simplificar los cálculos para obtener los parámetros de contacto: área real, carga normal y presión media, mediante subrutinas de solución del método cuadrado de Simpson mostrando que producen resultados aproximados con un mínimo error de $2.2 \%$. Los resultados muestran que es posible simplificar la modelación y simulación de casos de contacto más complejos y la determinación de otros parámetros de contacto.

\section{Descriptores}

- distribución Gauss

- distribución exponencial

- asperidades

- contacto elastoplástico 


\begin{abstract}
In order to model the elastic-plastic contact between rough surfaces, the exponential probabilistic distribution function of asperity heights, proposed by Greenwood and Williamson (1966), is analyzed as an approach of the Gaussian distribution function, and it is found to be impractical in most studied cases. The exponential probabilistic distribution modified function proposed by Polycarpou and Etsión (1999) is applied to the recent model of elastic-plastic contact of Kogut and Etsion (2004), to facilitate the calculations in obtaining the contact parameters (real area, normal load and mean pressure) using Simpson square method subroutines showing results with a minimum error in the order of $2.2 \%$. The results show that it is possible to simplify the modeling and simulation of more complex contact cases and the determination of other parameters of contact.
\end{abstract}

\section{Introducción}

El contacto elastoplástico entre dos superficies rugosas puede ser representado por una superficie rugosa y una lisa; que a su vez, parte de una esfera deformable y un plano rígido (Kougt y Etsion, 3003). Esta aproximación es un punto de partida para aplicaciones en aquellos componentes que entran en contacto superficial rugoso como: rodamientos, engranes y juntas homocinéticas, entre otras.

Hasta ahora, el tema de la topografía superficial sigue siendo de gran interés para varios investigadores como parte importante de mecánica del contacto, las interacciones superficiales y la fricción. Cuando dos superficies se unen, los contactos suceden en pequeñas áreas (Polycarpou y Etsion, 1999), debido a la rugosidad de ambas superficies. Al sumar todas esas pequeñas áreas se obtiene el área real de contacto de las superficies. La deformación comienza en donde sucede el contacto y puede ser elástica, elastoplástica o plástica, dependiendo de la presión nominal que sucede en el área real, la rugosidad superficial y las propiedades del material (Kogut y Etsion, 2004).

Varios investigadores han propuesto modelos de rugosidad superficial, partiendo de la suposición de una forma geométrica simple de las asperidades en la superficie con una distribución de probabilidad para los diferentes parámetros de las asperidades consideradas. El más común es el trabajo de Greenwood y Williamson (1966), donde consideran que la forma de la punta de las asperidades es esférica y de radio constante, y la altura de las asperidades está definida por una función probabilística de Gauss.

Greenwood y Williamson (1966), propusieron una función exponencial que se aleja grandemente de los valores obtenidos por la función probabilística de Gauss y, sobre todo, de las pruebas experimentales; ha- ciendo una revisión y análisis de algunas publicaciones se encontraron dos funciones exponenciales modificadas propuestas por Polycarpou (1999), que contemplan parámetros adicionales. Se utilizaron estas funciones en la determinación de los parámetros de contacto y se encontró en una de ellas una mejor aproximación con valores de error de 2.2, esto ayudó grandemente al cálculo simplificado y programado para la obtención de los parámetros de contacto como: área, carga normal, presión media, entre otros. También se logró la reducción de tiempo de corrida a la mitad; lo que antes se obtenía en 4 o 5 minutos se logró reducir a 2 o 3 minutos. Los resultados muestran que es posible simplificar la modelación y simulación de casos de contacto más complejos y la obtención de otros parámetros de contacto.

\section{Revisión bibliográfica}

El modelo de contacto básico "modelo basado en las asperidades", fue desarrollado por Greenwood y Williamson (GW) (1966). Este modelo ha sido la referencia de varios desarrollos para entender el comportamiento mecánico del contacto entre superficies metálicas rugosas. Desde el punto de vista estadístico, el contacto de algunas de las asperidades sobrepasará el límite elástico, aún para pequeñas cargas que comienzan a deformarse plásticamente (Pullen y Williamson, 1972).

Conforme la carga se incrementa, el número de asperidades que se deforman plásticamente, también se incrementa y el área correspondiente del modelo de GW (1966) se calcula según la teoría de Hertz, para la deformación elástica. Este modelo descarta la presencia de conservación del volumen en las regiones deformadas plásticamente (Mendenlson, 1968). El modelo básico de las asperidades de GW (1966), se extendió para superficies curvas por Greenwood y Tripp (GT) (1967), 
aún cuando GW ya habían hecho ciertos análisis, GT (1970-71) introdujeron dos superficies rugosas con asperidades desalineadas. Por otro lado, Hisakado (1974) consideró el radio variable de los picos de las asperidades.

Bush et al. (1975) analizaron el contacto con asperidades de forma elíptica paraboloide, Mc Cool (1986) consideró superficies anisotrópicas. Sin embargo, en todos estos modelos se despreció la conservación del volumen para las asperidades deformadas plásticamente. Francis (1977), reconoció el problema de la deformación plástica y estudió el rango de deformación de un micro-contacto al usar "funciones de conectividad continua", previamente determinadas en forma empírica por indentación esférica. Ishigaki et al. (1979) consideraron el problema de la deformación plástica.

Nayak (1971) presentó "un modelo de proceso aleatorio" bidimensional de una superficie rugosa y después con ello analizó el contacto plástico de superficies rugosas. Este modelo fue estimulado por las observaciones de Pullen y Williamson (PW) (1972), en el que el volumen es conservado por un incremento uniforme del contacto superficial, bajo ciertas restricciones específicas y cargas. El trabajo realizado por Kougut y Etsion (2002) contempla un modelo por elemento finito elástico-plástico de una esfera deformable y un plano rígido, como lo había trabajado Chang et al. (1987) para extenderlo más tarde al análisis del contacto en superficies rugosas.

Todos estos modelos determinan las expresiones del área de contacto, la carga normal de contacto y la presión media adimensional, considerando una distribución estadística de la altura de las asperidades y la altura de las superficies de Gauss. Otros estudios sobre el contacto de superficies rugosas incluyen la aplicación realizada en manejo de partículas (Vu Quoc et al., 2000), otros se basan en el contacto de una asperidad esférica (Bhushan, 1996), y en un modelo estadístico de múltiples asperidades de contacto (Bhusan, 1998).

Zhao et al. (2000) usaron una manipulación matemática para la transición lisa de las expresiones de la carga de contacto y el área de contacto entre el régimen de deformación elástica y plástica. Kuchasrski et al. (1994), resolvieron el problema de una esfera deformada por el método de elemento finito y desarrollaron expresiones proporcionales empíricas para la carga de contacto y el área de contacto. Así, otros trabajos sobre el contacto han sido realizados por elemento finito por Hardy et al. (1971), Kral et al. (1993), y Giannakopoulos (2000). Los resultados proporcionados por Mesarovic y Fleck (2000) para una esfera presionada por una placa y considerada como un espacio medio terminaron en el régimen plástico.

\section{Contacto elastoplástico en superficies rugosas con transición}

Para el análisis se considera una semiesfera deformable como se muestra en la figura 1 , con radio $R$ y presionada por una placa rígida. La línea punteada y continua en la esfera muestra la situación de antes y después de la deformación, respectivamente. Debido a la carga normal aplicada $\mathrm{P}$ se genera un aplastamiento denominado interferencia $\omega$ y un área de contacto de radio $a$ (Kogut y Etsion, 2004). La interferencia crítica $\omega_{c}$, marca la transición del régimen de deformación elástico al elastoplástico, como lo presenta Chang et al. (1987) y se define como:

$\omega_{c}=\left(\frac{\pi K H}{2 E}\right)^{2} R$

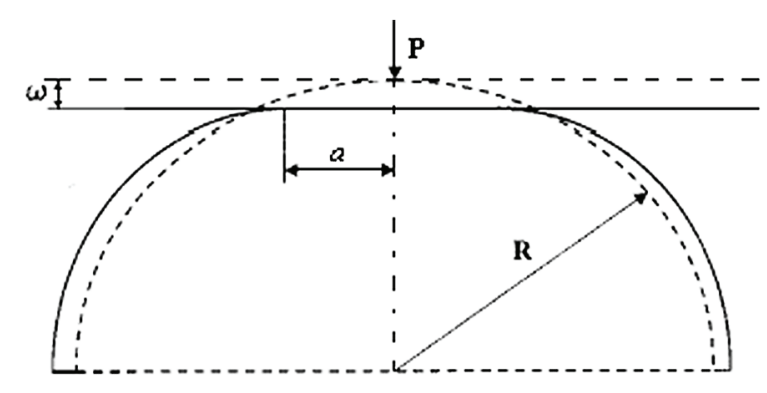

Figura 1. Deformación de una semiesfera presionada por una placa (Kogut y Etsion, 2004)

La dureza $\mathrm{H}$ está relacionada con la resistencia a la cedencia $\mathrm{Y}$ por $\mathrm{H}=2.8 \mathrm{Y}$ (Tabor, 1981). El coeficiente de dureza $\mathrm{K}$, está relacionado con la razón de Poisson $v$, descrita por Chang et al. (1987) como $\mathrm{K}=0.454+0.41 v$ y E es el módulo de elasticidad equivalente de los dos cuerpos en contacto evaluado conforme a Hertz (1881). Las zonas elástica, elastoplástica (con dos subregiones) y plástica fueron detalladamente determinadas mediante el método elemento finito en el trabajo de Etsion y Kogut (2003), donde el contacto se modeló mediante una asperidad representada por un cuarto de esfera, por la simetría y una placa considerada por una línea.

Durante la carga, el área de contacto $\bar{A}$ y la carga de contacto $\bar{P}$ de cada una de las asperidades, depende sólo de la interferencia $\omega$, suponiendo que no existe interacción entre las asperidades. La dependencia de $\bar{A}$ y $\bar{P}$ con $\omega$ debería estar determinada por el modo de deformación de la asperidad, el cual podría ser elástico, plástico o elastoplástico. El área y la carga de contacto total se obtienen al sumar las contribuciones de cada 
una de las asperidades como lo mostraron Greenwood y Williamson (1966) :

$$
\begin{aligned}
& A=\eta A_{n} \int_{d}^{\infty} \bar{A}(z-d) \varphi(z) d z \\
& P=\eta A_{n} \int_{d}^{\infty} \bar{P}(z-d) \varphi(z) d z
\end{aligned}
$$

donde:

$$
\begin{aligned}
\eta & =\text { la densidad de areal } \\
A_{n} & =\text { área normal, } \\
z & =\text { altura de las asperidades } \\
d= & \text { separación de la superficie plana y el plano } \\
& \text { medio de la altura de las asperidades y } \\
\varphi(z)= & \text { función de distribución probabilística. }
\end{aligned}
$$

La solución de Hertz para el contacto elástico de una esfera y un plano proporciona la carga de contacto $P_{e}$ y el área de contacto $A_{e}$, para $\omega \leq \omega_{c}$, en la forma:

$$
\begin{aligned}
& P_{e}=\frac{4}{3} E R^{1 / 2} \omega^{3 / 2}=P_{c}\left(\frac{\omega}{\omega_{c}}\right)^{3 / 2} \\
& A_{e}=\pi R \omega=A_{c}\left(\frac{\omega}{\omega_{c}}\right)
\end{aligned}
$$

Donde $P_{c}$ y $A_{c}$ son la carga normal y el área de contacto, respectivamente en la interferencia crítica $\omega=\omega_{c}$. $P$ y $A$ quedan normalizadas por $P_{c}$ y $A_{c}$ respectivamente, para obtener la función de la interferencia adimensional $\omega / \omega_{c}$. Estas funciones son independientes de las propiedades del material y del radio de la esfera.

$$
\begin{aligned}
& A_{c}=\pi R \omega_{c} \\
& P_{c}=\frac{4}{3} E R^{1 / 2} \omega_{c}^{3 / 2}
\end{aligned}
$$

Usando las ecuaciones (1), (4) y (5), la presión media $p_{e}=3 / 2 P_{e} / A_{e}$ según Hertz (1881) para $\omega \leq \omega_{c}$ es:

$$
p_{e}=p_{c}\left(\frac{\omega}{\omega_{c}}\right)^{1 / 2}
$$

donde $p_{e}$ es la presión de contacto media y $p_{c}$ la presión de contacto normal en $\omega=\omega_{c}$.

Para $\omega>\omega_{c}$ el contacto es elastoplástico y se requiere una solución numérica para encontrar la relación entre $\omega / \omega_{c}$, la carga, el área y la presión media de contacto. Etsión y Kogut (2003), usaron el método de elemento finito para obtener la solución numérica, donde el contacto entre la esfera y plano presentan diferentes fases de deformación, elástica, transición elastoplastica y plástica. Ellos también adoptaron el criterio de cedencia de von Mises, para determinar la transición de la deformación elástica a la plástica, que coincide con los resultados experimentales realizados por Bushan (1996).

La solución del problema fue mediante una semiesfera deformable y se modeló como un cuarto de disco suponiendo una simetría de revolución. El plano rígido se modeló como una línea y el material de la esfera se supuso con comportamiento elástico perfectamente plástico similar a tensión y compresión. Para generalizar la solución presente y eliminar la necesidad de una entrada específica, los resultados numéricos, se normalizaron con respecto al valor crítico correspondiente en la iniciación de la falla, $\omega_{c}$, de manera similar que en las ecuaciones anteriores. La normalización de la presión de contacto medio $p_{m}$, se realizó con respecto a la resistencia a la cedencia del material, $Y$, de la esfera del material.

La validez de esta normalización se probó resolviendo el problema con varios materiales diferentes en el rango de $100<E / Y<1000, v=0.3$, y la esfera de radio en el rango $0.1 \mathrm{~mm}<R<10 \mathrm{~mm}$. Los resultados adimensionales $P / P_{c}, A / A_{c}$. y $p_{m} / Y$ contra la interferencia adimensional $\omega / \omega_{c}$ fueron siempre los mismos, a pesar de la selección de las propiedades del material y el radio de esfera.

El modelo numérico se verificó primero con los resultados analíticos de la solución de Hertz, en el régimen elástico para $\omega<\omega_{c}$. La verificación incluye la carga, el radio del área de contacto y la distribución de esfuerzos en el área de contacto, a lo largo del eje de simetría. La diferencia entre los resultados numéricos y analíticos siempre fue menor de $2.8 \%$. Otra verificación fue con el modelo de Kogut y Etsion (2003), en el régimen elástico plástico (para $1<\omega / \omega_{c}<110$ ), al incrementar la densidad del mallado. Las más grandes diferencias en la carga y el área de contacto fueron de 1 y $3 \%$, respectivamente.

Kogut y Etsion (2003), encontraron que la superficie de la esfera de la región de contacto es dividida en tres subregiones:

I) Una subregión elástica circular interna, extendiéndose radialmente del centro del contacto hasta el filo del núcleo elástico.

II) Una subregión intermedia anular, entre el filo del núcleo elástico y el frente más externo de la región plástica.

III) Una subregión elástica posterior (figura 2). 
La evolución del contacto elastoplástico puede dividirse en tres etapas distintas. La primera para $1 \leq \omega / \omega_{c} \leq 6$, donde la región plástica se desarrolla por debajo de la superficie de la esfera y la entrada del área es elástica. La segunda para $6 \leq \omega / \omega_{c} \leq 110$, donde el área es elástica plástica conteniendo una subregión plástica anular, confinada por la parte interna y externa de la elástica. La tercera etapa para $\omega / \omega_{c}>110$ que corresponde al área de contacto plástico.

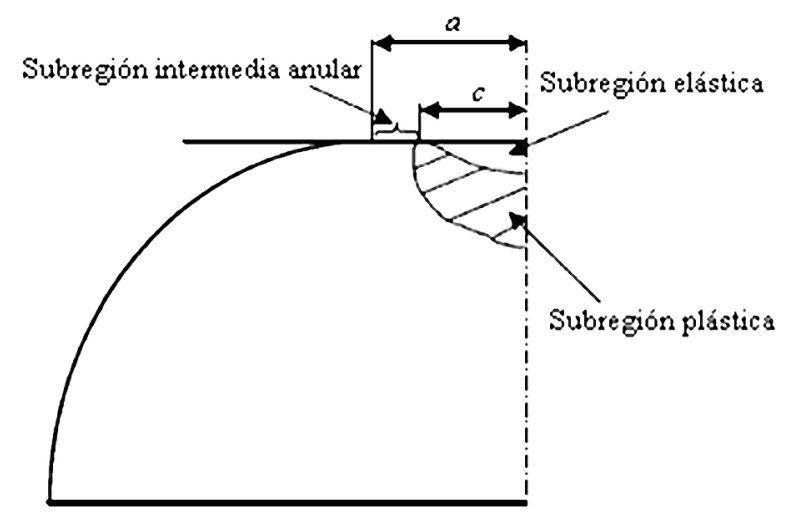

Figura 2. Subregiones en la zona de contacto (Kogut y Etsion, 2003)

Kogut y Etsion (2003), encontraron que el régimen de contacto elástico plástico de una asperidad, se extendía en el rango de $1 \leq \omega / \omega_{c}<110$ con una transición $\omega / \omega_{c}=6$, es decir, se divide en dos subregiones. Los parámetros adimensionales de contacto de área, carga normal y presión media presentados en las ecuaciones (4), (5) y (8), respectivamente, pueden expresarse en la forma:

$$
\begin{aligned}
& \frac{A}{A_{c}}=d\left(\frac{\omega}{\omega_{c}}\right)^{e} \\
& \frac{P}{P_{c}}=a\left(\frac{\omega}{\omega_{c}}\right)^{b}
\end{aligned}
$$

Las constantes $a, b, d$ y $e$ son valores que se determinaron por un análisis de elemento finito en las diferentes zonas de deformación elástico, elastoplástico (con las dos subregiones de transición) y plástico como se muestran en la tabla 1, éstas se basan en la suposición de que el material es elástico perfectamente plástico (Kogut y Etsion, 2003).
Tabla 1. Valores de $a, b, d$ y e para varios regímenes de deformación (Kogut y Etsion, 2003)

\begin{tabular}{lcccc}
\hline & \multicolumn{4}{c}{ Constantes } \\
\cline { 2 - 5 } Régimen de deformación & $a$ & $b$ & $d$ & $e$ \\
\hline $\begin{array}{l}\text { Elástico } \omega / \omega_{c}<1 \\
\text { 1er elástico plástico }\end{array}$ & 1 & 1.5 & 1 & 1 \\
$\begin{array}{l}1 \leq \omega / \omega_{c} \leq 6 \\
\text { 2a elástico plástico }\end{array}$ & 1.03 & 1.425 & 0.93 & 1.136 \\
$\begin{array}{l}6 \leq \omega / \omega_{c} \leq 110 \\
\text { Plástico } 110>\omega / \omega_{c}\end{array}$ & $3 / \mathrm{k}$ & 1 & 2 & 1 \\
\hline
\end{tabular}

Posteriormente, las constantes se usaron para el cálculo de los parámetros área $A^{*}$ y carga $P^{*}$ de contacto adimensional entre dos superficies rugosas, en las zonas de deformación elástico, elastoplástico (con las dos subregiones de transición) y plástico mostradas en cada porción de las siguientes ecuaciones como:

$$
\begin{aligned}
A^{*}=\frac{A}{A_{n}}=\pi \beta \omega_{c}^{*}\left(\int_{d^{*}}^{d^{*}+\omega_{c}^{*}} I^{1}+0.93 \int_{d^{*}+\omega_{c}^{*}}^{d^{*}+6 \omega_{c}^{*}} I^{1.136}+\right. \\
\left.0.94 \int_{d^{*}+6 \omega_{c}^{*}}^{d^{*}+110 \omega_{c}^{*}} I^{1.146}+2 \int_{d^{*}+110 \omega_{c}^{*}}^{\infty} I^{1}\right)
\end{aligned}
$$

donde:

$\beta=R A_{n^{\prime}}$

$\eta \quad$ es la densidad areal,

$R$ es el radio de las asperidades,

$A_{n}$ es el área nominal de la superficie,

$I_{e}$ es la forma general del integrando para $A$,

y se tiene entonces:

$$
\begin{aligned}
& I^{e}=\left(\frac{z^{*}-d^{*}}{\omega_{c}^{*}}\right)^{e} \varphi^{*}\left(z^{*}\right) d z^{*} \\
& P^{*}=\frac{P}{A_{n} H}=\frac{2}{3} \pi \beta K \omega_{c}^{*}\left(\int_{d^{*}}^{d^{*}+\omega_{c}^{*}} I^{1.5}+1.03 \int_{d^{*}+\omega_{c}^{*}}^{d^{*}+6 \omega_{c}^{*}} I^{1.425}+\right. \\
& \left.1.4 \int_{d^{*}+6 \omega_{c}^{*}}^{d^{*}+110 \omega_{c}^{c}} I^{1.263}+\frac{3}{k} \int_{d^{*}+110 \omega_{c}^{\infty}}^{\infty} I^{1}\right)
\end{aligned}
$$

donde $I^{b}$ es la forma general del integrando para $P$, y se tiene entonces:

$I^{b}=\left(\frac{z^{*}-d^{*}}{\omega_{c}^{*}}\right)^{b} \varphi^{*}\left(z^{*}\right) d z^{*}$ 


\section{Funciones de distribución de altura de las asperidades}

Los resultados experimentales obtenidos por Greenwood y Williamson (1996) demostraron que varias de las superficies siguen una aproximación de la distribución de Gauss de la altura de las asperidades. Se tiene entonces:

$\varphi^{*}(z)=\frac{1}{\sqrt{(2 \pi)}} e^{-\frac{1}{2} z^{2}}$

Además propusieron una distribución estadística exponencial de la altura de las asperidades para simplificar el análisis de las ecuaciones anteriores, como:

$$
\varphi^{*}\left(z^{*}\right)=e^{z^{*}}
$$

Resultando muy robusta y alejada de los valores calculados para los parámetros de contacto. Polycarpou y Etsion (2004) encontraron también que la distribución exponencial que propusieron Greenwood y Williamson (1966) es inadecuada para la mayoría de los casos. Ellos mismos sugirieron una aproximación exponencial que garantiza algo muy cercano a la distribución de Gauss para determinar el área normal, la carga normal y el número de asperidades en contacto, aplicada en el contacto elastoplástico propuesto por Chang et al. (1987).

Como la suposición de la distribución de Gauss representa cierto proceso aleatorio para el caso de la altura de las asperidades bastante adecuado, Polycarpou y Etsion se dieron a la tarea de encontrar los coeficientes para la función exponencial modificada, para aproximar la función de distribución de Gauss, al menos en el rango de interés del análisis de contacto. Esto se realizó mediante la aproximación de mínimos cuadrados directamente sobre la función de densidad probabilística, resultando:

$\varphi_{1}^{*}\left(z^{*}\right)=1.3 e^{-1.6 z^{*}}$

$\varphi_{2}^{*}\left(z^{*}\right)=17 e^{-3 z^{*}}$

En seguida, se presentan las simulaciones para obtener los parámetros de contacto normal (área real, carga normal y presión media) para compararse junto con los valores obtenidos por Kogut y Etsion (2003).

\section{Análisis de resultados}

A continuación se presentan las comparaciones de las aproximaciones aplicadas al modelo de Kogut y Etsion (2003) para determinar los parámetros de área real, carga de contacto, y presión media usados en artículos de Greengood y Williamson (1996), Chang et al. (1987), entre otros, como se muestran en las gráficas que a continuación se presentarán en unidades adimensionales.

Para las simulaciones de carga normal contra separación adimensional (distancia entre el plano medio de la altura de las asperidades y la superficie lisa), se usaron integrales que se resolvieron numéricamente empleando el software MatLab, con la subrutina de solución del método cuadrado de Simpson. La figura 3 muestra los resultados de variación de separación adimensional contra carga normal adimensional, para funciones de distribución de altura de las superficies de Gauss, la simple exponencial de Greenwood y Williamson (1966), exponencial modificada 1, exponencial modificada 2 y según las ecuaciones (15), (16), (17) y (18), respectivamente.

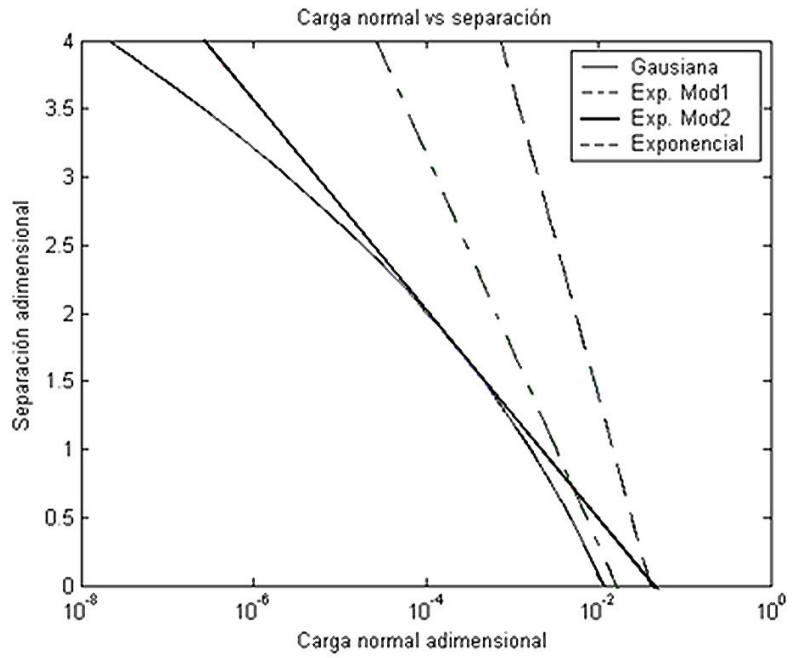

Figura 3. Carga normal contra separación

Se consideraron los valores originales usados por Kogut y Etsion (2003) muy parecidos a los usados por Greengood y Williamson (1966) y Chang et al. (1987), en donde $\eta=0.004 \times 10^{12}, R=110 \times 10^{-6}, \sigma_{a}=100 \times 10^{-9}, H=7$ $\times 10^{9}, v=0.3, E_{1,2}=207 \times 109$ y $\psi=0.5 ;$ y la variación de valores de separación adimensional en el rango de 0 a 4 con incrementos de 0.1 . 


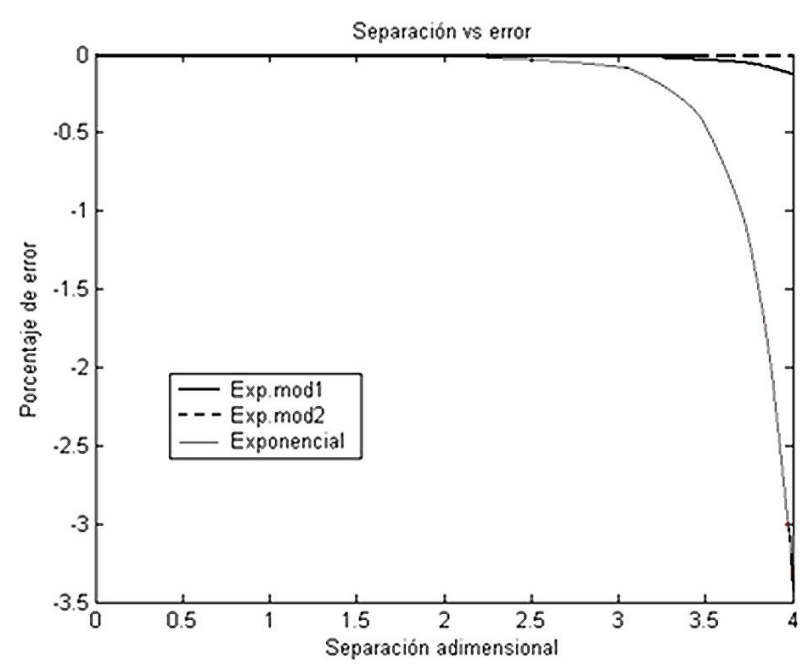

Figura 4. Gráfica de error de comparación de las distibuciones

Como se muestra en la gráfica de la figura 3 , los valores de separación disminuyen conforme la carga normal aumenta, la línea que más se aproxima a la distribución de Gauss es la exponencial modificada 2, los valores de la exponencial modificada 1 se disparan conforme aumenta la separación, en el caso de la función la exponencial simple propuesta por Greenwood y Williamson (1966), los valores están demasiado alejados.

Si se comparan los resultados de la función distribución de Gauss punto por punto y se grafican, podrá observarse en la figura 4 las funciones resultantes del error de aproximación con las distribuciones exponencial simple, exponencial modificada 1 y exponencial modificada 2. Efectivamente el error se dispara en la exponencial simple. Estas comparaciones se realizaron en el rango de separación de 0-4, resultando errores bajos insignificantes para el caso de las funciones de distribución modificadas propuestas, sobre todo, para la exponencial modificada 2.

Detallando un poco más los resultados, la gráfica de la figura 5 muestra la función de error de aproximación de la exponencial modificada 2 con la función de distribución de Gauss, donde los errores máximos alcanzan valores absolutos de 2.2 y se mantienen muy parecidos en el rango de valores de la separación entre 1 y 2.5, para valores mayores de 3 se incrementan significativamente.

Al igual que Polycarpou y Etsion (1999), se compararon resultados para la distribución de Gauss de las superficies contra la exponencial modificada 1 y 2 y la simple, propuesta por Greenwoood y Williamson (1966). La que más se acercó a los valores obtenidos por la distribución de Gauss fue la exponencial modificada 2

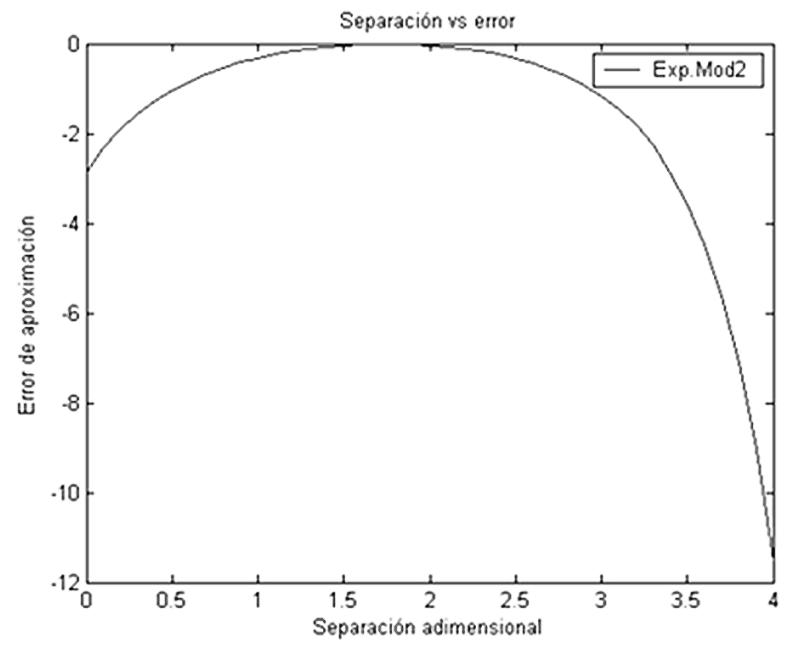

Figura 5. Error de comparación distribución Gauss y exponencial 2

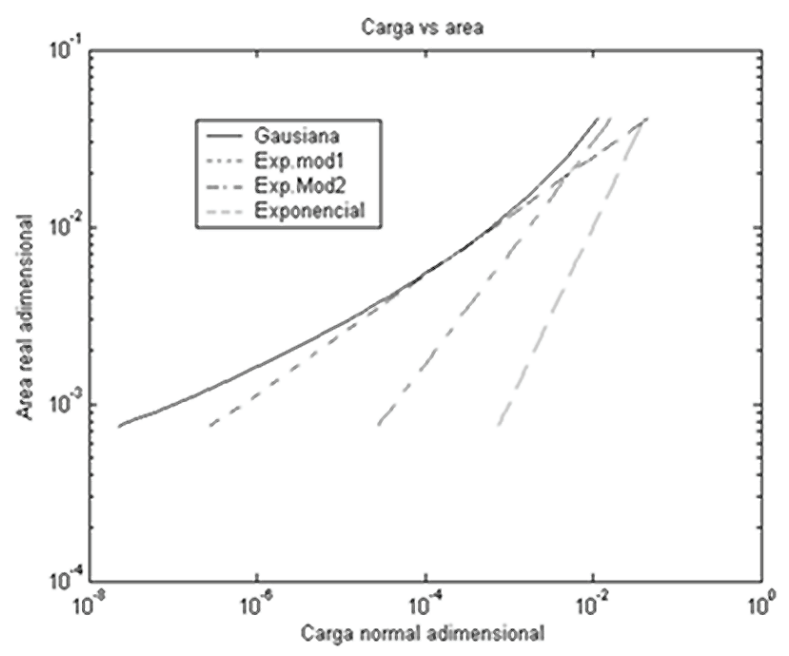

Figura 6. Carga normal contra área real elástico plástico de E-K

para determinar la carga de contacto en contacto elastoplástico propuesta por Kogut y Etsion (2004), la aproximación fue hasta de $12 \%$ y disminuye conforme aumenta la separación.

De manera parecida al caso anterior, se realizó el análisis de variación del área de contacto normal contra la carga normal de contacto adimensional, comparando los valores obtenidos de la función de distribución de Gauss, contra la exponencial simple, la exponencial modificada 1 y la exponencial modificada 2. Se observa que la carga es proporcional al área, excepto para la distribución de Gauss de la altura de las superficies, y que la función de distribución exponencial modificada 2 se aproxima bastante bien a los 


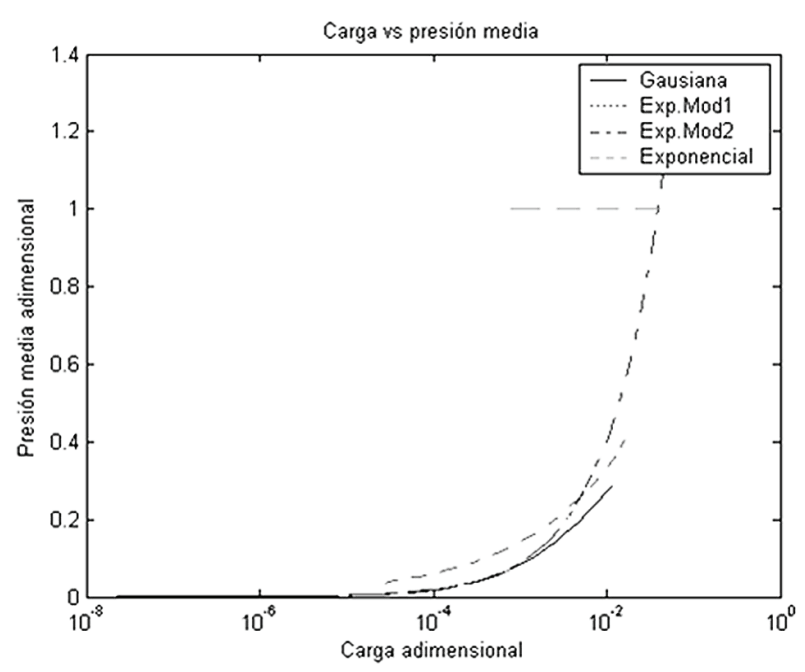

Figura 7. Carga contra presión media elástica plástica K-E

valores de la distribución de Gauss e iguala en algunos valores del rango de $10^{-4}$ a $10^{-3}$.

Las líneas de la figura 7 muestran la variación de la presión media contra la carga normal adimensional, para los valores presentados anteriormente, considerando también distribución de Gauss, la exponencial modificada 1, la exponencial modificada 2 y la exponencial simple.

La aproximación obtenida de la distribución exponencial modificada 2 es idéntica en cierto rango de valores, mientras que para la exponencial simple, se pierden por completo los valores obtenidos.

\section{Conclusiones}

Se demostró que la función de distribución probabilística exponencial simple de la altura de las asperidades para modelar el contacto de superficies rugosas propuesta por Greenwood y Williamson (1966) es impráctica para la mayoría de las aplicaciones, ya que los valores de los parámetros de contacto de área real, carga normal y presión media varían grandemente con los obtenidos por la distribución probabilística de Gauss.

Los resultados muestran que las aproximaciones de distribución probabilística exponencial modificada propuesta por Polycarpou y Etsion (1999), son idénticos a los obtenidos con el trabajo reciente realizado por Kogut y Etsion (2003) con la distribución probabilística de Gauss, conteniendo errores mínimos.

Se realizaron las simulaciones con el software numérico de MatLab, usando las ecuaciones obtenidas por Kogut y Etsión (2003) para determinar los parámetros de contacto como área real, carga normal y presión media; mediante subrutinas de solución del método cuadrado de Simpson se encontró que efectivamente la distribución probabilística exponencial modificada de la altura de las asperidades propuesta por Polycarpou, simplificó mucho los cálculos y los resultados obtenidos.

Los resultados muestran que es posible simplificar la modelación y simulación de casos de contacto más complejos y la obtención de otros parámetros de contacto como la fuerza de adhesión y la carga tangencial en la interfase, para determinar los coeficientes de rozamiento estático y dinámico, según sea el caso; usando la distribución probabilística exponencial modificada de la altura de las asperidades para simplificar los cálculos y análisis de la mecánica del contacto.

\section{Referencias}

Bush A.W., Gibson R.D., Thomas T.R. The Elastic Contact of Rough Surface. Wear, volumen 35, 1975: 87-111.

Bhushan B. Contact Mechanics of Rough Surfaces in Tribology: Single Asperity Contact. Appl. Mech. Rev., volumen 49 (número 5), 1996: 275-298.

Bhushan B. Contact Mechanics of Rough Surfaces in Tribology: Multiple Asperity Contact. Tribol. Lett., volumen 4 (número 1), 1998: 1-35.

Chang W.R., Etsion I., Bogy D.B. An Elastic-Plastic Model for the Contact of Rough Surfaces. ASME Journal Tribology, volumen 109, 1987: 257-263.

Francis H.A. Application of Spherical Indentation Mechanics to Reversible and Irreversible Contact between Rough Surfaces. Wear, volumen 45 (número 2), 1977: 221-269.

Giannakopoulos A.E. Strength Análysis of Spherical Indentation of Piezoelectric Materials. ASME J. Appl. Mech., volumen 67, 2000: 409-416.

Greengood J.A., Tripp J.H. The Elasctic Contac of Rough Spheres. ASME Journal of Appl. Mech., volumen 34, 1967: 153-159.

Greengood J.A., Tripp J.H. The Contac of Two Nominally Flat Rough Surfaces. Proc. Instn. Mech. Engrs, volumen 185, 19701971: 625-633.

Greenwood J.A., Williamson J.B.P. Contact of Nominally Flat Surfaces. Proc. Roy. Soc, Londres, Series. A., volumen 295 (número 1442), 1966: 300-319.

Hardy C., Baronet C.N., Tordion G.V. The Elastoplastic Indentation of a Half-Space by a Rigid Sphere. Int. J. Numer. Methods Engs., volumen 3, 1971: 451-462.

Hertz H., J. Reine Angew, Math., volumen 92, (número 156), 1881. Hisakado T. Effects of Surface Roughness on Contact between Solid Surfaces. Wear, volumen 28, 1974: 217- 234. 
Ishigaki H., Kawaguchi I., Mizuta S. A Simple Estimation of the Elastic-Plastic Deformation Asperities. Wear, volumen 54, 1979: 157-164.

Kogut E.I. Elastic-Plastic Contact Analysis of a Sphere and a Rigid Flat. ASME Journal Appl. Mech., volumen 69, 2002: 657-662.

Kogut L., Etsion I. A Finite Element Based Elastic-Plastic Model for the Contact of Rough Surfaces. Tribol. Trans., volumen 46, 2003: 383-390.

Kogut L., Etsion I. A Static Friction Model for Elastic-Plastic Contacting Rough Surfaces. ASME Journal of Tribology, volumen 126 (número 1), 2004: 34-40.

Kral E.R., Komvoupolus K., Bogy D.B. Elástic-Plastic Finite Element Analysis of Repeated Indentation of Half-Space y a Rigid Sphere. ASME Journal Appl. Mech., volumen 60 (número 4) 1993: 829-841.

Kuchasrski S., Klimezak T., Polijaniuk A., Kaczmarek J. Finite Element Model for the Contact of Rough Surfaces. Wear, volumen 177, 1994: 1-13.

Mendelson A. Plasticity: Theory and application, 1968.

Mesarovic S.D., Fleck N.A. Frictionless Indentation of Disimilar Elasctic-Plastic Spheres. Int. J. Solid Struct., volumen 37, 2000: 7071-7091.

McCool J.I. Predicting Microfracture in Ceramic Via a Microcontac Model. ASME Journal of Tribology, volumen 108, 1986: 380386.

Nayak P.R. Random Process Model Of Rough Surfaces. ASME Journal of Lubrication of Technology, volumen 93, 1971: 398-407.

Polycarpou A.A., Etsion I. Analytical Approximations in Modeling Contacting Rough Surfaces. ASME Journal of Tribology, volumen 121, 1999: 234-239.

Pullen F., Williamson F.B.P. On the Plastic Contact of Rough Surfaces. Proc. Roy. Soc., London, Ser. A., 327, 1972: 59-173.
Tabor D. Friction-The Present State of our Understanding. ASME Journal Lubr. Technol., volumen 103, 1981: 169- 179.

Vu-Wuoc L., Zhang X., Lesburg L. A Normal Force-Displacement Model for Contacting Sphere Accounting for Plastic Deformation: Force-Driven Formulation. ASME J. Appl. Mech., volumen 67, 2000: 363-371.

Zhao Y., Mietta D.M., Chang L. An Asperity Microcontact Model Incorporation the Transition from Elastic Deformation to Fully Plastic Flow. ASME Journal Tribology, volumen 122, 2000: 86-93.

\section{Este artículo se cita:}

\section{Citación Chicago}

Sánchez-Rodríguez, Álvaro, José Salvador Echeverría-Villagómez, Raúl Lesso-Arrollo, Francisco Javier García-Rodríguez, Roberto Salas-Zuñiga. Contacto elastoplástico entre superficies rugosas con distribución de Gauss y exponencial. Ingeniería Investigación y Tecnología, XIII, 03 (2012): 317-326.

\section{Citación ISO 690}

Sánchez-Rodríguez A., Echeverría-Villagómez J.S., LessoArrollo R., García-Rodríguez F.J., Salas-Zuñiga R. Contacto elastoplástico entre superficies rugosas con distribución de Gauss y exponencial. Ingeniería Investigación y Tecnología, volumen XIII (número 3), julio-septiembre 2012: 317-326. 


\section{Semblanza de los autores}

Álvaro Sánchez-Rodríguez. Recibió el grado de maestro en ingeniería mecánica por el Instituto Tecnológico de Celaya, Guanajuato, México. Es coordinador de la maestría en ingeniería mecánica en el Instituto Tecnológico de Celaya. Actualmente es profesor en el Departamento de Ingeniería Mecánica del Instituto Tecnológico de Celaya y candidato al doctorado en el campo de ingeniería del Centro de Ingeniería y Desarrollo Industrial (CIDESI) Qro., México. Ha publicado 12 artículos en congresos nacionales e internacionales.

José Salvador Echeverría-Villagómez. Recibió el grado de maestro en ingeniería mecánica por la Universidad Nacional Autónoma de México (UNAM). Actualmente es director de Metrología Física del Centro Nacional de Metrología (CENAM) y profesor en el Departamento de Ingeniería Mecánica del Instituto Tecnológico de Celaya. Es doctor en filosofía en el campo de ingeniería por la Universidad de Cambridge. Ha publicado más de 100 artículos en revistas y congresos nacionales e internacionales.

Raúl Lesso-Arroyo. Recibió el grado de maestro en ingeniería mecánica por la Universidad de Guanajuato (FIMEE), Guanajuato, México. Actualmente es profesor en el Departamento de Ingeniería Mecánica del Instituto Tecnológico de Celaya. Ha publicado más 60 artículos en revistas y congresos nacionales e internacionales. Ha desarrollado más de 25 proyectos para empresas y centros de investigación.

Francisco Javier García-Rodríguez. Recibió el grado de maestro en materiales por el Centro de Investigación y de Estudios Avanzados del Instituto Politécnico Nacional (Cinvestav), México. Trabaja como profesor en el Departamento de Mecánica del Instituto Tecnológico de Celaya. Es doctor en ingeniería de materiales por el Centro de Investigación y de Estudios Avanzados del Instituto Politécnico Nacional (Cinvestav). Ha publicado más de 40 artículos en revistas nacionales e internacionales.

Roberto Salas-Zuñiga. Recibió el grado de maestro en ingeniería eléctrica por el Centro de Investigación y de Estudios Avanzados del Instituto Politécnico Nacional (Cinvestav), México. Actualmente trabaja como consultor independiente y ha sido profesor en diversas instituciones como: ITEMS, UAQ, CINVESTV. Es doctor en el campo de la ingeniería eléctrica por el Centro de Investigación y de Estudios Avanzados del Instituto Politécnico Nacional (Cinvestav).Ha publicado más de 30 artículos en revistas y congresos nacionales e internacionales. 